\title{
Do physical therapy interventions improve urinary incontinence and quality of life in patient with multiple sclerosis: A systematic literature review
}

\author{
Najwa Alfarra*, Hala Aldosary and Sultan Almefleh \\ Department of physical therapy, King Faisal Specialist Hospital and Research Center, Kingdom of Saudi Arabia
}

\begin{abstract}
Background: Multiple sclerosis (MS) presents with many symptoms, including urinary incontinence (UI) that physical therapy can play very important role, which is widely prevent, but the physical therapy management for UI in MS population lacks consensus. We analyzed the current evidence for effectiveness of physical therapy to decrease UI and improve quality of life (QoL) in population with MS.

Purpose: To systematically review the literature and present the best available evidence for the efficacy and effectiveness of physical therapy intervention in treating the urinary incontinence for MS population and improve QoL.

Data source: PubMed, Cochrane library, BMJ Group, BioMed Central, Wiley online library, Cumulative Index to Nursing and Allied Health Literature, and PEDro. Study selection: 5 randomized, control trials (RCTs) and one clinical trial published in English from 2006- May 2019.

Data extraction: Any study concentrated on surgical or pharmaceutical treatment interventions, focused on bowel incontinence or were not within the physical therapy scope of practice.

Data synthesis: The study focus on physical therapy intervention for MS patients with UI. and randomized control study.

Limitation of the study: The reviewed study is limited to 6 randomized control trial.

Conclusion: There is significant evidence that physical therapy interventions in MS patients with urinary incontinence are very effective and had significant change in reducing UI and increasing QoL.
\end{abstract}

\section{Introduction}

Multiple sclerosis (MS) is the second most frequent cause of disability in young people, after public way accidents. MS is a chronic, progressive neurological disease involving the deterioration of the white matter pathways in the brain and spinal cord. MS is generally described as either relapsing -remitting, characterized by deterioration of the myelin and associated neural axons [1].

The symptoms vary depending on the location of the lesion in the central nervous system and disease progression type, leading to subsequent multiple physical disabilities. Common impairments include fatigue, ataxia, tremor, spasticity, bladder or bowel dysfunction, impaired vision, pain, cognitive disorders, dysfunctions, dysphagia, and sexual dysfunction $[1,2]$.

Bladder dysfunction is highly prevalent and affected approximately $80 \%-100 \%$ of MS patients through the course of disease [3]. $60 \%$ to $80 \%$ of patients show overactive bladder (OAB) caused by parasympathetic dysfunction due to brain and spinal cord damage [4]. Other bladder disorders include impaired detrusor contractility in $20 \%$ of patients, due to hypotonic bladder, and lack of coordination in $25 \%$ of patients, due to detrusor-sphincter dyssinergia, leading to voiding dysfunctions, incomplete emptying, or urinary retention $[5,6]$.
The term of urinary incontinence includes both stress and urge incontinence. Stress incontinence occurs when the pelvic floor muscles are too weak to stop urine from leaking when coughing, laughing, or sneezing. Urge incontinence or overactive bladder occurs when urine leakage is closely preceded by a powerful urge to pass urine [7].

There are several specific diagnostics - therapeutic protocols for MS and specialized protocols or guidelines for patients with any neurogenic bladder dysfunction such as anti-cholinergic drugs, botulinum toxin, surgical intervention, and physical therapy intervention such as pelvic floor muscle training (PFMT), developed by Dr. Kegel in 1948 for use in stress urinary incontinence. The rational is to the strength of the pelvic floor muscles and to prevent adverse perineal movement during intra-abdominal pressure changes $[8,9]$. Neuromuscular electrical stimulation (NMES) and electromyography (EMG) biofeedback are another physical therapy modality that teach

${ }^{\star}$ Correspondence to: Najwa Alfarra, Department of physical therapy, King Faisal Specialist Hospital and Research Center, Kingdom of Saudi Arabia, E-mail: mohm@hotmail.com

Key words: quality of life questionnaires, pelvic floor exercises, physical therapy, bladder dysfunction, urinary incontinence, neurogenic bladder, multiple sclerosis Received: July 16, 2019; Accepted: July 25, 2019; Published: August 02, 2019 
the patient to control voluntary muscle relaxation and contraction through visual and auditory feedback. Physical therapy intervention has proved to be effective in reducing/cured the urinary incontinence in non-multiple sclerosis population, but very few literatures evaluated physical therapy intervention for the treatment of patients with MS $[10,11]$.

These symptoms are not life threating, and thus are often neglected by healthcare professionals. However, bladder dysfunction is responsible for a significant negative impact on the quality of life (QoL) of affected patients [12]. The embarrassing nature of urinary incontinence affects patients QOL in many ways, as measured by using the 36-item short form health status survey [12]. The greatest impacts are seen in the physical and social functioning, emotional health, and role limitations. Also, urinary incontinence was associated with depression and low self-esteem along with reduced activities of daily living [12]. The purpose of this study was to review the existing literature regarding the effectiveness of $\mathrm{PT}$ in reducing, urinary incontinence and increasing quality of life in people with MS.

\section{Objective}

This review aimed to identify the benefits of physical therapy for urinary incontinence in patients with multiple sclerosis and to verify the effect of urinary incontinence on the patient's quality of life.

\section{Methodology}

\section{Methods}

Systematic review of the literature was performed through electronic search from December 21, 2017. By Identifying the studies from PubMed, Cumulative Index to Nursing and Allied Health Literature, PEDro, Cochrane library, BMJ Group, and Wiley online library databases for the 2006-May 2019 period of time. The references of retained articles were considered and articles responding to inclusion criteria but not present in the initial search were selected. The keywords used were either in combination or independently: quality of life questionnaires, pelvic floor exercises, physical therapy, bladder dysfunction, urinary incontinence, Neurogenic bladder, multiple sclerosis.

\section{Inclusion and exclusion criteria}

The inclusion of articles in this review was based on the following criteria: clinical trials and randomized controlled studies concerning adult subjects and written in English, as well as literature reviews were used, participants had a diagnosis of MS with either stress incontinence, urgency or overactive bladder, the intervention performed by the physical therapist focusing on urinary incontinence, the outcome measures included QOL assessed before and after intervention. The exclusion criteria any study concentrated on surgical or pharmaceutical treatment interventions, focused on bowel incontinence or were not within the physical therapy scope of practice.

\section{Study selection}

Three investigators decided on study eligibility according to recommendations from the Scottish intercollegiate Guideline developer handbook for systematic reviews of interventions to include original publications of randomized controlled trial, clinical trials, as well as literature reviews published in English from 2006-May 2019. Full tests of the RCTs, and clinical trials that examine the impact of physical therapy intervention on urinary incontinence in MS patients. The study excluded secondary data analysis, case reports, case series, and RCTs that did not report patient outcomes.
Primary researcher (N.A) independently perform a fist selection of articles based on abstracts to retain articles dealing with physical therapy intervention in MS patients. A recursive search of the references from relevant articles was completed. Articles were evaluated by a pair of reviewers (H.A., S.A.) and verified that the selected articles met the criteria.

\section{Assessment of methodological quality}

The quality of study was analyzed by using the following criteria: participant selection, length and loss of follow-up, use of intentionto-treat principle, masking of the treatment status, randomization shame, adequacy of randomization and allocation concealment, and justification of sample size. Several strategies were used to reduce bias, including a comprehensive literature search for published evidence in several databases, a search of reference lists of systematic reviews and proceeding of the International Continence Society. The quality of the selected studies was assessed using a standard grading system, as Scottish Intercollegiate guideline network [13]. Evidence table cab be found at (Appendix 1 and 2).

\section{Data synthesis}

Forty-five articles were selected from electronic bibliographies and screened for retrieval $(n=45)$. Thirty-one articles were excluded for not meeting the selection criteria $(n=31)$ such as intervention not PT based or case report or includes bowel incontinence, or no full texts available. The resultant was fourteen randomized control trials and clinical trials full articles $(n=14)$. Eight articles were exempted for not meeting the inclusion criteria $(n=8)$ such as not specific to MS and incontinence. The six most appropriate articles were left $(n=6)$. Two of them were systematic review and were used as a background reference because they did not report any statistical data (Figure 1).

Summaries of the studies included in the review are provided in (Table 1). Studies are presented the information about the level of evidence, population, interventions investigated, outcome measures and information of determine the generalizability of the study findings (Table 2).

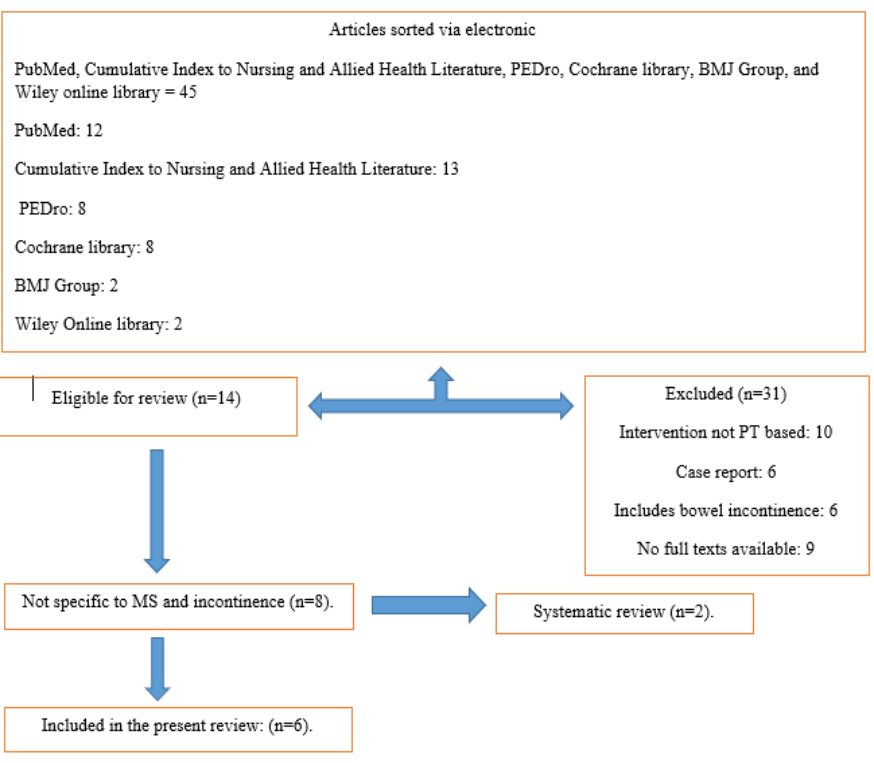

Figure 1. Results of search 
Table 1. Comparative summary of best evidence

Considered judgment table

Key question:

Do Physical Therapy Interventions Improve Urinary Incontinence and Quality of Life in patient with Multiple Sclerosis?

1.Quality of evidence:

six studies have surveyed the significance of physical therapy interventions in treating urinary incontinence and improve QOL in MS population. All the studies were of good quality methodologically and have reduction in urinary incontinence and improvement in QOL.

2. Applicability:

The evidence is fully applicable as it shows physical therapy intervention reduces existing urinary incontinence as well as significantly improved QOL in MS population.

3. External validity:

It is reasonable to generalize the results of all the 6 studies in the target population as the integrity of the studies is safeguarded and a sizeable randomized sample of the population with similar characteristics used.

4 Consistency:

There is a high degree of consistency in the available evidence. There is no study that demonstrated conflicting results.

5. Quantity of evidence:

All the studies included had evidence that was statistically significant and with significant impact in reduction of urinary incontinence and improved QOL.

6. Clinical impact:

Physical therapy interventions if implemented both correctly and consistently will have a great impact in urinary incontinence reduction and improve QOL in MS population. There are no indicated risks of the intervention in the evidence available.

\section{Other factors:}

There were no other factors taken into consideration when assessing evidence base.

\section{Evidence statement:}

Patients with MS usually experience urinary incontinence in the course of their disease, with great impact on their QOL. Physical Evidence level

therapy interventions can reduce the urinary incontinence and increase QOL. Treatment protocols for the management of UI in $1++$

patient with MS is an integral part of the physical therapist daily practice and should be a part of rehabilitation program for the MS 1+

patients.

9.Reccommendation:

MS patients should receive extensive physical therapy protocol to reduce urinary incontinence and increase QOL. B

Table 2. Evidence table (Mackway K, et al.)

\begin{tabular}{|c|c|c|c|c|c|c|}
\hline Bibliographic citation & $\begin{array}{l}\text { Study type } \\
\text { \& Evi. Lev }\end{array}$ & Population & Intervention & Follow-up time & Outcome measures & Effect \\
\hline $\begin{array}{c}\text { Forough F, } \\
\text { Moosa S, Habib S, Payam S, } \\
\text { Mahnaz S (2017) Pelvic floor } \\
\text { muscle training instruction to } \\
\text { control urinary incontinence } \\
\text { and its resulting, anxiety } \\
\text { and depression in patients } \\
\text { with multiple sclerosis. } \\
\text { Jundishapur J Chronic Dis } \\
\text { Care. } 2017\end{array}$ & Clinical trial & $\begin{array}{c}50 \text { female patients } \\
\text { with MS }\end{array}$ & PFMT & Three months & $\begin{array}{c}\text { Incontinence } \\
\text { questionnaires-urinary } \\
\text { incontinence short form } \\
\text { (ICIQ-UI-SF) } \\
\text { 21-Item depression, } \\
\text { anxiety and stress scale } \\
\text { (DASS-21) } \\
\text { Both used before and after } \\
\text { the intervention }\end{array}$ & $\begin{array}{l}\text { PFMT was effective } \\
\text { in reducing UI and its } \\
\text { resulting stress, anxiety } \\
\text { and depression in patient } \\
\text { with MS }\end{array}$ \\
\hline \multicolumn{7}{|c|}{ General Comments: The study design is very good. The study findings can be generalized to the MS population. The study gives verified figures with significant P-Values. } \\
\hline $\begin{array}{c}\text { Lucio AC, } \\
\text { Perissionto MC, Natalin } \\
\text { RA, Prudente A, Damasceno } \\
\text { BP, Dancona CA (2011) } \\
\text { Comparative study of pelvic } \\
\text { floor muscle training in } \\
\text { women with MS: its impact } \\
\text { on lower urinary tract } \\
\text { symptoms and quality of life. } \\
\text { Clinics } 2011 \text {. }\end{array}$ & $\begin{array}{c}\text { RCTs } \\
++\end{array}$ & $\begin{array}{c}35 \text { women with MS and } \\
\text { urinary incontinence } \\
\text { Treatment group } 18, \\
\text { sham group } 17\end{array}$ & \begin{tabular}{|c|} 
Pelvic floor muscle \\
training (PFMT) with \\
assistance from vaginal \\
perineometer and \\
instructions to practice the \\
exercise daily at home, \\
versus perineometer \\
inside the vagina with no \\
exercise required. Twice / \\
week for 30 minutes
\end{tabular} & Three months & $\begin{array}{c}\text { Incontinence } \\
\text { questionnaires-urinary } \\
\text { incontinence short form } \\
\text { (ICIQ-UI-SF) } \\
\text { Overactive bladder } \\
\text { questionnaire } \\
\text { QoL }\end{array}$ & $\begin{array}{l}\text { There was improvement } \\
\text { in the Quality of life and } \\
\text { reduction of overactive } \\
\text { bladder symptoms for } \\
\text { the women who did the } \\
\text { PFMT. }\end{array}$ \\
\hline
\end{tabular}

General Comments: The study is good designed. There is reduction in the overactive bladder symptoms with intervention in the control group. There is treatment integrity and inter observer agreement. but there is no proof that the women actually continued with PFMT as trained at home. Results in scientific terms with P-Value well evaluated. It can be generalized

\begin{tabular}{|c|c|c|c|c|}
\hline $\begin{array}{l}\text { McClurg D, Ashe RG, Lowe- } \\
\text { Strong AS (2009) Electrical } \\
\text { Stimulation is a useful } \\
\text { adjunct in the management } \\
\text { of urinary incontinence } \\
\text { in people with multiple } \\
\text { sclerosis. Australian Journal } \\
\text { of physiotherapy. }\end{array}$ & $\begin{array}{c}\text { RCTs } \\
++\end{array}$ & $\begin{array}{l}74 \text { females with MS. } \\
\text { Treatment group } 37, \\
\text { sham group } 37\end{array}$ & $\begin{array}{c}\text { Both groups were } \\
\text { taught skills and } \\
\text { strategies to prevent } \\
\text { incontinence and trained } \\
\text { in pelvic floor muscle } \\
\text { exercises. Both received } \\
\text { electrical stimulation } \\
\text { at either vaginal or } \\
\text { anal, the treatment } \\
\text { group received active } \\
\text { stimulation while the } \\
\text { control group received } \\
\text { sham stimulation } \\
\text { performed the exercise } \\
\text { daily. }\end{array}$ & $\begin{array}{l}24 \text { weeks } \\
\text { The exercises were reviewed } \\
\text { with electromyography } \\
\text { biofeedback at weekly clinic } \\
\text { visit. }\end{array}$ \\
\hline
\end{tabular}

group had significantly less incontinence, with 0.8 fewer $\begin{array}{ll}\text { episode per day) } & \text { incontinence, with } 0.8 \text { fewer } \\ \text { episodes/day }(95 \% \text { CI } 0.1\end{array}$ Pad weight. Symptoms
to 1.4 ) and $89 \%$ lighter pads questionnaires, pelvic $\quad(95 \%$ CI 8 TO 1.71) than floor muscle function the control group. At 24 \begin{tabular}{l|c} 
using Oxford, and EMG. & the control group. At 24 \\
weeks, pad weights were
\end{tabular} Outcomes were measured the only objective outcome at 9,16, and 24 weeks. the only objective outcom significant.

General Comments: Study Design is very good. Results in scientific terms with P-Value and confidence intervals well evaluated. Can be generalized to the MS population. 


\begin{tabular}{|c|c|c|c|c|c|c|}
\hline $\begin{array}{c}\text { Khan F, Pallant JF, Pallant } \\
\text { JI, Brand C, Kilpatrick } \\
\text { TJ, (2010) A randomized } \\
\text { controlled trial: outcomes } \\
\text { of bladder rehabilitation } \\
\text { in persons with multiple } \\
\text { sclerosis. J Neural Neurosur } \\
\text { Psychiatry. }\end{array}$ & $\begin{array}{c}\text { RCTs } \\
++\end{array}$ & $\begin{array}{l}58 \text { women with MS, } \\
\text { treatment group }(n=34), \\
\text { control }(n=24)\end{array}$ & $\begin{array}{l}\text { Treatment group } \\
\text { received personalized, } \\
\text { multidisciplinary } \\
\text { rehabilitation program } \\
2-3 / \text { week for } 6 \\
\text { weeks. continue with } \\
\text { maintenance program for } \\
\text { twelve months. Control } \\
\text { group maintenance } \\
\text { program only }\end{array}$ & $\begin{array}{c}12 \\
\text { months }\end{array}$ & IIQ-7, UDI-6, QOL & $\begin{array}{l}\text { The treatment group } \\
\text { compared with the } \\
\text { control group showed } \\
\text { improvement: } 78 \% v s . \\
27 \% \text { for UDI6 and } 59 \% \\
\text { vs. } 17 \% \text { improved for } \\
\text { IIQ7. More patients } \\
\text { in the control group } \\
\text { deteriorated over the } \\
\text { study period on the } \\
\text { UDI6 ( } 30 \% \text { vs. } 0 \% \text {; } \\
\text { p }<0.001 \text { ) and IIQ7 ( } 39 \\
\text { vs. } 0 \% ; \mathrm{p}=0.001) \text {. }\end{array}$ \\
\hline \multicolumn{7}{|c|}{$\begin{array}{l}\text { General Comments: The study invalid as the result of improvements were made by subjective observation, and the researcher did not allocate specific interventions to be used for the } \\
\text { bladder dysfunction, the results are applicable since the study is randomized, and subject are women with MS and incontinence. It can be generalized to MS women population. }\end{array}$} \\
\hline \begin{tabular}{|c|} 
McClurg D, Ashe RG, \\
Mashall K, Lowe- \\
Strong AS (2006) \\
Comparison of pelvic \\
floor muscle training, \\
electromyography \\
biofeedback, and \\
neuromuscular electrical \\
stimulation for bladder \\
dysfunction in people \\
with multiple sclerosis: a \\
randomized pilot study. \\
Neurourol Urodyn. 2006
\end{tabular} & $\begin{array}{c}\text { RCTs } \\
++\end{array}$ & 30 women with MS. & $\begin{array}{c}\text { Three groups } \\
\text { 1-Pelvic Floor Training } \\
\text { and Advice (PFTA), } \\
\text { 2-PFTA, and EMG } \\
\text { Biofeedback, 3-PFTA, } \\
\text { EMG Biofeedback and } \\
\text { Neuromuscular electrical } \\
\text { stimulation } \\
\text { (NMES) }\end{array}$ & 9 weeks & $\begin{array}{l}\text { 24-hour pad test, digital } \\
\text { assessment of pelvic } \\
\text { floor, EMG, QOL } \\
\text { questionnaire (QII and } \\
\text { UDI) }\end{array}$ & $\begin{array}{l}\text { Group } 3 \text { demonstrated } \\
\text { superior benefit as } \\
\text { measured by the number } \\
\text { of leaks and pad test } \\
\text { than Group 2, with } \\
\text { Group } 1 \text { showing less } \\
\text { improvement when } \\
\text { compared to week } 0 ; \\
\text { this was statistically } \\
\text { significant between } \\
\text { Groups } 1 \text { and } 3 \text { for } \\
\text { number of leaks } \\
(\mathrm{P}=0.014) \text { and pad tests } \\
(\mathrm{P}=0.001) \text {, and Groups } \\
1 \text { and } 2 \text { for pad tests } \\
(\mathrm{P}=0.001) \text {. A similar } \\
\text { pattern was evident for all } \\
\text { other outcome measures. }\end{array}$ \\
\hline \multicolumn{7}{|c|}{$\begin{array}{l}\text { General Comments: The study is strongly designed, but no indication of treatment integrity. Results collected by standard scientific tool so no bias, with confidence intervals and } \\
\text { significant P-Values. The result could be generalized for MS population. }\end{array}$} \\
\hline \begin{tabular}{|c|} 
Deseze M, Raibaut P, \\
Gallien P. et al (2011) \\
Transcutaneous posterior \\
tibial nerve stimulation \\
for treatment of the \\
overactive bladder \\
syndrome in multiple \\
sclerosis: result of a \\
multicenter prospective \\
study. Neurourol Urodyn. \\
2011 \\
\end{tabular} & Cohort & $\begin{array}{l}70 \text { patients, } 51 \text { women } \\
\text { and } 19 \text { men }\end{array}$ & $\begin{array}{c}\text { Transcutaneous posterior } \\
\text { tibial nerve stimulation } \\
\text { (TPTNS) }\end{array}$ & 3 months & QOL questionnaires & $\begin{array}{c}\text { Clinical improvement } \\
\text { of OAB was shown in } \\
82.6 \% \text { and } 83.3 \% \text { of } \\
\text { the patients on D30 and } \\
\text { D90, respectively, with } \\
\text { significant improvement } \\
\text { of primary and secondary } \\
\text { outcomes compared to } \\
\text { baseline }\end{array}$ \\
\hline \multicolumn{7}{|c|}{ Comments: good quality, there is treatment integrity. Applicable to the MS population } \\
\hline
\end{tabular}

\section{Data analysis}

Seven RCTs ( $\mathrm{n}=7$ ) were included. All studies reported adequacy of randomization, discussed participant selection, length and loss of follow up, use of intention-to-treat principle, and masking of the treatment status for both subjects and investigators. All the studies reported adequate allocation concealment. There are marked heterogeneity in the type and intensity of interventions in both groups. All the studies used validated measurement tools.

Quality of life participation level was performed by all the studies. Analysis of incontinence recorded (urgency and leakage per $24 \mathrm{~h}$ ) was performed using data from Forough et al., Lucio et al., McClure et al., and De Seze et al.

Forough $\mathrm{F}$ reported significant improvement in MS patients with incontinence, who participated in the PFMT compared with control group, like the ICIQ-UI-SF score and frequency of urine leakage decreased significantly after the intervention compared to pre-intervention $(\mathrm{P}<0.001)$. after the intervention, urine leakage disappeared completely in less than one fourth of patients $(n=12)$ and decreased to once a week in almost half of the patients $(42.2 \%)$. The results revealed that the mean score of stress $(\mathrm{P}<0.001)$, anxiety $(\mathrm{P}=0.04)$ and depression $(\mathrm{P}=0.003)$ significantly decreased with three months of pelvic floor muscle exercise and reduced urinary incontinence [14].

Lucio reported a statistical significant reduction between the groups in the following: Overactive assessment $(\mathrm{P}<0.0001)$, ICIQ$\mathrm{SF}$ assessment $(\mathrm{P}=0.0003)$, the Specific Impact of Urinary Problems on Quality of Life (SIUP) domain $(\mathrm{P}=0.0001)$, and the general QoL domain $(\mathrm{P}=0.0443)$, after 24 sessions. While, there is no difference in SF-36 assessment were found between the groups $[15,16]$.

McClurg study showed that the treatment group had less incontinence, with 0.8 fewer episodes/day (95\% CI 0.1 TO 1.4) and $89 \mathrm{~g}$. lighter pads (95\% CI 8 to 171) than the control group. Symptoms were also rated as significantly less bothersome. So, the electrical stimulation and using of biofeedback improved the urinary incontinence. The researcher had certain concern about the nature of the disease (MS is characterized by period of relapse and remission. So, it is not clear whether the treatment benefits of the 9-weeks intervention used in this study would persist across these periods [11].

Khan, et al. [17] the researchers found that the treatment group compared with the control group showed improvement: $78 \%$ vs. $27 \%$ for UDI-6 and 59\% vs. 17\% improved for IIQ-7. More patients in the 
control group deteriorated over the study period on the UDI- $6(30 \%$ vs. $0 \% ; \mathrm{p}<0.001)$ and IIQ-7 (39 vs. $0 \% ; \mathrm{p}=0.001)$ he suggested the Individualized bladder rehabilitation program reduces disability and improves QoL in patient with MS.

McClurg et al. the authors concluded that the combination of PFTA, EMG, biofeedback, and NMES can reduce urinary incontinence symptoms in people with MS.

Deseze et al. [8] found that there is clinical improvement of $\mathrm{OAB}$ was shown in $82.6 \%$ and $83.3 \%$ of the patients on D30 and D90, respectively, with significant improvement of primary and secondary outcomes compared to baseline.

\section{Discussion}

The aim of this systematic review was to determine whether the current literature supports the impact of physical therapy as a treatment for urinary incontinence and QOL in people with MS. Six studies met the inclusion criteria and all the studies showed statistical significance for the incontinence leakage episodes and QOL improvement. So, it confirms the evidence of Pelvic Floor Muscle Training (PFMT) Neuromuscular Electrical Stimulation (NMES), EMG biofeedback, and Transcutaneous Posterior Tibial Nerve Stimulation (TPTNS) intervention in the treatment of urinary incontinence in MS patients from full text studies published in English during the last 13 years.

Overall, these results allow the physiotherapist to reject the null hypothesis that physical therapy intervention do not improve QOL or UI in patients with MS. The quality of most of the RCTs was good; participants were not excluded from the analysis of outcomes, and randomized was adequate. However, allocation concealment was not addressed in two studies. Variations in outcome measures rather than RCT quality, resulted in heterogeneity between studies. The measurement of outcomes was consistent across the studies. Difference in the numbers of participants, types of interventions may have affected the results, and the duration of treatment the longer duration will result in greater effect. Additionally, different modalities of treatment will give different result.

Despite extensive efforts to standardize outcome assessment for urinary incontinence. The included RCTs measured a variety of outcomes, including adherence to PFMT, self-reported symptoms, signs, and improvement; severity of urinary incontinence as assessed by pad weights specific and quality of life questionnaires. Another factor which may influence outcome is the degree to which subjects actually comply with the treatment program prescribed and adhered to the PFMT. Subject compliance or adherence was infrequently and generally poorly reported with no standardized, validated or reliable approach to its assessment.

Physical therapy interventions in the included studies resulted in a direct effect on incontinence leakage episodes. The moderate effect sizes in QOL measures may indirectly result from fewer leakage episodes, but multiple factors can contribute to change in QOL. For example, fatigue, muscle weakness, time required for ADL's, and depression can result in a decrease in QOL participation and activity levels. Change in any of these factors may contribute to improvement noticed in this sample of participants. Furthermore, the decrease in urinary incontinence can reduce fatigue and depression, which will impact the effect of QOL.

\section{Implications for practice}

The findings of the selected studies suggest that PFMT, NMES and EMG brings about better outcomes as compared to non-treatment for treating urinary incontinence in MS population. In the cases where PFMT was used, the women were more likely to experience improvement or get cured entirely [18-20]. These women also reported fewer leakage episodes per day, better quality of life, and have less urine leakage on short pad tests as compared to non-treatment.

Most of the selected studies imply that treatment, especially in self-reported cases, has a greater impact for MS patient with urinary incontinence taking part in a closely monitored PFMT programme for no less than three months [18]. Additionally, age does not matter can, therefore, not reduce the effect of treatment in urinary incontinent women.

The selected studies imply suggest that the treatment effect is magnified if the PFMT programme is focused on valid psychological principles. For a successful programme, the right contraction has to be confirmed and recorded before the training, and the participants are monitored and supported to continue with the programme [21]. There is an overall widespread endorsement among the selected studies that PFMT should be integrated into the first line conservative management programmes for MS population with urinary incontinence.

However, most of the selected studies lack follow up past the completion of the treatment programme. Therefore, it would be difficult to establish the long-term results from the application of PFMT [22]. Additionally, design and conduct pelvic floor muscle training program for patients with progressive neurological disorders is difficult because of the evolving nature of the disease that may result in short-lived improvement due to progression of the disease and the development of refractory symptoms. Regardless, some of the studies hold that long-term outcomes of PFMT are significantly greater when the participants are supervised for no less than three months. If the participant continues with the programme for an extended period, the treatment effect is likely to be enhanced accordingly or at least remain constant.

\section{Limitation of the study}

There are several limitations in this study that may have affected the results. The sample sizes for individual studies were small, which decrease the power of each study, but the grand effect sizes for most of the outcomes were significant when the studies were compared. Most of the studies lacked a long-term follow-up. The studies included more than physical therapy modalities which restrict the ability of the physical therapist to identify specific interventions that deliver the greatest effects. All authors used SF-36 which assess general QoL, so in this literature the questionnaire was not adequate sensitive to detect the specific measurement that impact the urinary incontinence, frequency, and noctoria. So, these questions provide important information about the effects of treatment and measure the rehabilitation outcomes and the patient's evaluation of their own health.

\section{Conclusion}

Overall, there is evidence for the widespread recommendation for use of pelvic floor muscle training either in combination with EMG, NMES or without in preventing and treating urinary incontinence for MS population as compared to non-intervention. The limited nature of follow-up beyond the end of treatment in the majority of the published studies means that the long-term effects may be greater in MS participating in supervised PFMT for at least three months. Continued adherence to training may be associated with maintained or increased treatment effect, but this hypothesis needs further testing. There is a need for at least one large, well conducted, and explicitly 
reported randomized trial, comparing physical therapy intervention with a control to investigate the longer-term clinical effectiveness of PFMT in MS population.

In conclusion, physical therapy interventions are beneficial and have no significant adverse effects. Substantially and durable improvements in continence and QOL can be achieved, when the patient is appropriately selected, and the exercises are adequately performed.

\section{References}

1. Nicholas R, Rashid W (2013) Multiple sclerosis. Am Fam Physician 87: 712-714. [Crossref]

2. Coote S, Hogan N, Franklin S (2013) Falls in people with multiple sclerosis who use a walking aid: prevalence, factors, and effect of strength and balance interventions. Arch Phys Med Rehabil 94: 616-621. [Crossref]

3. Minardi D, Muzzonigro G (2005) Lower urinary tract and bowel disorders and multiple sclerosis: role of sacral neuromodulation: a preliminary report. Neuromodulation 8: 176-181. [Crossref]

4. Fingerman JS, Finkelstein LH (2000) The overactive bladder in multiple sclerosis. $J$ Am Osteopath Assoc 100: 9-12. [Crossref]

5. Courtney AM, Castro-Borrero W, Davis SL, Frohman TC, Frohman EM (2011) Functional Treatments In Multiple Sclerosis. Curr Opin Neurol 24: 250-254. [Crossref]

6. Fjorback MV, Van Rey FS, Rijkhoff NJM, Nøhr M, Petersen T, et al. (2007) Electrical Stimulation Of Sacral Dermatomes In Multiple Sclerosis Patients With Neurogenic Detrusor Overactivity. Neurourol Urodyn 26: 525-530. [Crossref]

7. National Institute for Health and Care Excellence (2013) Urinary incontinence: introduction: a general introduction to urinary incontinence.

8. De Sèze M, Raibaut P, Gallien P, Even-Schneider A, Denys P, et al. (2011) Transcutaneous posterior tibial nerve stimulation for treatment of the overactive bladder syndrome in multiple sclerosis: results of a multicenter prospective study. Neurourol Urodyn 30: 306-311. [Crossref]

9. Dumoulin C, Hay-Smith EJ, Mac Habée-Séguin G (2010) Pelvic floor muscle training versus no treatment, or inactive control treatment, for urinary incontinence in women. Cochrane Database Syst Rev 5: CD005654. [Crossref]

10. De Ridder D, Vermeulen C, Ketelaer P, Van Poppel H, Baert L (1999) Pelvic floor rehabilitation in multiple sclerosis. Acta Neurol Belg 99: 61-64. [Crossref]
11. McClurg D, Ashe RG, Marshall K, Lowe-Strong AS (2006) Comparison of pelvic floor muscle training electromyography biofeedback, and neuromuscular electrical stimulation for bladder dysfunction in people with multiple sclerosis: a randomized pilot study. Neurourol Urodyn 25: 337-348. [Crossref]

12. D'Ancona CA, Tamanini JT, Botega N, Lavoura N, Ferreira R, et al. (2009) Quality of life of neurogenic patients: translation and validation of the Portuguese version of Qualiveen. Int Urol Nephrol 41:29-33. [Crossref]

13. SIGN (2012) Scotish intercollegiate guideline network. A guideline developer handbook.

14. Forough F, Moosa S, Habib S, Payam S, Mahnaz S (2017) Pelvic floor muscle training instruction to control urinary incontinence and its resulting, anxiety and depression in patients with multiple sclerosis. Jundishapur J Chronic Dis Care 6: e37333.

15. Milsom I, Abrams P, Cardozo L, Roberts RG, Thüroff J, et al. (2001) How widespread are the symptoms of an overactive bladder and how are they managed? a populationbased prevalence study. BJU Int 87: 760-766. [Crossref]

16. Lúcio AC, Perissinoto MC, Natalin RA, Prudente A, Damasceno BP, et al. (2011) A comparative study of pelvic floor muscle training in women with multiple sclerosis: its impact on lower urinary tract symptoms and quality of life. Clinics (Sao Paulo) 66: 1563-1568. [Crossref]

17. Khan F, Pallant JF, Pallant JI, Brand C, Kilpatrick TJ (2010) A randomised controlled trial: outcomes of bladder rehabilitation in persons with multiple sclerosis. $J$ Neurol Neurosurg Psychiatry 81: 1033-1038. [Crossref]

18. Dumoulin C, Glazener C, Jenkinson D (2011) Determining the optimal pelvic floor muscle training regimen for women with stress urinary incontinence. Neurourol Urodyn 30: 746-753. [Crossref]

19. Dannecker C, Wolf V, Raab R, Hepp H, Anthuber C (2005) EMG-biofeedback assisted pelvic floor muscle training is an effective therapy of stress urinary or mixed incontinence: a 7-year experience with 390 patients. Arch Gynecol Obstet 273: 93-97. [Crossref]

20. Reilly ET, Freeman RM, Waterfied MR, Waterfield AE, Steggles P, et al. (2002) Prevention of postpartum stress incontinence in primigravidae with increased bladde neck mobility: a randomised controlled trial of antenatal pelvic floor exercises. BJOG 109: 68-76 [Crossref]

21. Aqur WI, Steggles P, Waterfield M, Freeman RM (2008) The long-term effectiveness of antenatal pelvic floor muscle training; 8-year follow up of a randomized controlled trial. BJOG 115: 985-990. [Crossref]

22. Tubaro A (2004) Defining overactive bladder: epidemiology and burden of disease Urology 64: 2-6. [Crossref]

Copyright: (C2019 Alfarra N. This is an open-access article distributed under the terms of the Creative Commons Attribution License, which permits unrestricted use, distribution, and reproduction in any medium, provided the original author and source are credited. 